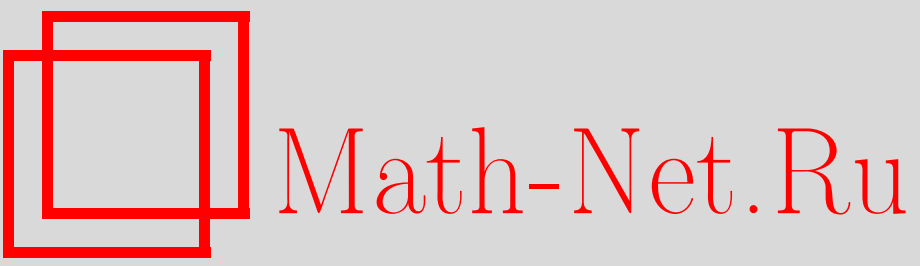

И. А. Чельцов, Метод вырождения и нерациональность трехмерных многообразий с пучком поверхностей дель Пеццо, УМН, 2004, том 59, выпуск 4, 203-204

DOI: https://doi.org/10.4213/rm768

Использование Общероссийского математического портала Math-Net.Ru подразумевает, что вы прочитали и согласны с пользовательским соглашением

http://www.mathnet.ru/rus/agreement

Параметры загрузки:

IP: 52.205.19.152

26 апреля 2023 г., 18:09:43 


\title{
МЕТОД ВЫРОЖДДЕИЯ И НЕРАЦИОНАЛЬНОСТЬ ТРЕХМЕРНЫХ МНОГООБРАЗИЙ С ПУЧКОМ ПОВЕРХНОСТЕЙ ДЕЛЬ ПЕЦЦО
}

\author{
И. А. ЧЕЛЬцов
}

Проблема рациональности алгебраических многообразий является одной из наиболее глубоких и интересных проблем алгебраической геометрии. Глобалшные голоморфные диффференциалшные формы являются естественными бирационалњными инвариантами гладкого многообразия, которые полностью решают проблему рациональности алгебраических кривых и поверхностей (см. [1]). Однако уже в трехмерном случае существуют нерациональные многообразия, которые во многом очень близки к рациональным, и имеющихся дискретных инвариантов уже не хватает для определения рационалшности. Например, существуют трехмерные нерациональные, но унирациональные многообразия (см. [1] и [2]). Тем не менее, получить подобные примеры нелегко. На данньй момент существует четыре способа доказательства нерациональности рационально связных многообразий (см. [2]). В настоящей работе мы дадим простое доказательство следующего хорошо известного результата.

ТеОрема 1. Пусть $X \subset \mathbb{P}^{1} \times \mathbb{P}^{3}$ - подмногообразие, являющееся общим әффективным дивизором бистепени $(m, 3)$, а $V$ - двулистное накрытие $\mathbb{P}^{1} \times \mathbb{P}^{2}$ с ветвлением в общем дивизоре бистепени $(m, 4)$. Тогда оба многообразия $V$ и $X$ нерациональны при $m \geqslant 2$.

Отметим, что многообразие $X$ в теореме 1 представимо в виде расслоения на кубические поверхности, а $V$ - в виде расслоения на поверхности дель Пеццо степени 2 . Известно, что многообразия $X$ и $V$ унирациональны, а при $m \leqslant 1$ рациональны. Нерациональность $V$ при $m=2$ следует из [3]. Нерациональность $X$ при $m \geqslant 2$ доказана в [4]. Бирациональная жесткость и нерациональность $X$ и $V$ при $m \geqslant 3$ доказана в [5]. Из [1] и [6] следует нерациональность $X$ при $m \geqslant 5$ и нерациональность $V$ при $m \geqslant 2$. Все структуры расслоений Мори на многообразии $X$ описаны в [7] в случае $m=2$, откуда также следует его нерационалшность.

Для доказательства теоремы 1 мы воспользуемся следующим результатом в [8], современное доказательство которого содержится в [1] и который обобщает стандартную технику вырождения (см. [2]).

ТеОРема 2. Пусть $\xi: Y \rightarrow Z-$ собственный плоский морфизм с неприводимыми и приведенными слоями. Тогда существует такое счетное множество замкнутых подмногообразий $Z_{i} \subset Z$, что для любой замкнутой точки $s \in Z$ слой $\xi^{-1}(s)$ является линейчатым многообразием, если и только если $s \in \bigcup Z_{i}$.

Возьмем прямую $L \subset \mathbb{P}^{3}$ и точку $O \in \mathbb{P}^{2}$. Рассмотрим гладкую поверхность $S=\alpha^{-1}(L)$ и гладкую кривую $C=\beta^{-1}(O)$, где $\alpha: \mathbb{P}^{1} \times \mathbb{P}^{3} \rightarrow \mathbb{P}^{3}$ и $\beta: \mathbb{P}^{1} \times \mathbb{P}^{2} \rightarrow \mathbb{P}^{2}$ - проекции на второй множитель. Пусть $\widehat{X} \subset \mathbb{P}^{1} \times \mathbb{P}^{3}$ - общий эффективный дивизор бистепени $(m, 3)$, содержащий поверхность $S$, а $\pi: \widehat{V} \rightarrow \mathbb{P}^{1} \times \mathbb{P}^{2}$ - двулистное накрытие с ветвлением в достаточно общем дивизоре $D$ бистепени $(m, 4)$, имеющем особенность типа $\mathbb{A}_{1} \times \mathbb{C}$ в общей точке кривой $C$.

ЗАмечАниЕ 3. Многообразия $\widehat{X}$ и $\widehat{V}$ могут быть получены как плоские собственные деформации многообразий $X$ и $V$ соответственно. Более того, оба многообразия $\widehat{X}$ и $\widehat{V}$ рационально связны (см. [1]). С другой стороны, рационально связное трехмерное многообразие линейчато, если и только если оно рационально. Таким образом, из теоремш 2 следует, что для доказательства теоремы 1 достаточно доказать нерациональность $\widehat{X}$ и $\widehat{V}$ при $m \geqslant 2$.

Геометрически построенное вырождение означает следующее. Общий слой естественной проекции $X$ и $V$ на $\mathbb{P}^{1}$ - гладкая поверхность дель Пещо степени 3 и 2 соответственно, определенная

Автор очень признателен М. М. Гриненко, В.А. Исковских, С. А. Кудрявцеву, Ю. Г. Прохорову, А.В. Пухликову и В.В. Шокурову за плодотворные беседы. Все рассматриваемые многообразия считаются проективными и определенными над полем $\mathbb{C}$. 
над полем $\mathbb{C}(x)$, которая имеет группу Пикара $\mathbb{Z}$ при $m \geqslant 1$. С другой стороны, общий слой проекции $\widehat{X}$ на $\mathbb{P}^{1}$ является гладкой кубической поверхностью в $\mathbb{P}^{3}$, которая содержит прямую. Проекция из этой прямой задает на многообразии $\widehat{X}$ структуру расслоения на коники. Общий слой проекции $\widehat{V}$ на $\mathbb{P}^{1}$ является поверхностью дель Пеццо степени 2 , которая имеет одну обькновенную двойную точку. Пучок антиканонических кривых на этой поверхности, проходящих через двойную точку, задает на $\widehat{V}$ структуру расслоения на коники.

Пусть $\gamma: U \rightarrow \mathbb{P}^{1} \times \mathbb{P}^{3}$ - раздутие поверхности $S \cong \mathbb{P}^{1} \times \mathbb{P}^{1}$, а $\chi: W \rightarrow \mathbb{P}^{1} \times \mathbb{P}^{2}$ - раздутие кривой $C$. Положим $\widetilde{X}=\gamma^{-1}(\widehat{X})$ и $\widetilde{D}=\chi^{-1}(D)$. Пусть $\widetilde{\pi}: \widetilde{V} \rightarrow W-$ двулистное накрытие с ветвлением в дивизоре $\widetilde{D} \subset W$. Локалшные вычисления показывают, что $\widetilde{X}$ и $\widetilde{V}$ неособы в силу общности выбора дивизоров $\widehat{X}$ и $D$. Отметим, что по построению $\widetilde{X}$ и $\widetilde{V}$ являются разрешением особенностей многообразий $\widehat{X}$ и $\widehat{V}$ соответственно. Причем $\widehat{X}$ обязательно особо при $m \geqslant 1$, но оно всегда нодально в силу общности выбора $\widehat{X}$, а $\left.\gamma\right|_{\widetilde{X}}$-малое разрешение.

Проекция из прямой $L$ индуцирует морфизм $\tau: \widetilde{X} \rightarrow \mathbb{P}^{1} \times \mathbb{P}^{1}$, а проекция из точки $O$ индуцирует морфизмы $\eta: \widetilde{V} \rightarrow \mathbb{P}^{1} \times \mathbb{P}^{1}$. По построению, морфизмы $\tau$ и $\eta$ являются расслоениями на коники. Причем $\operatorname{Pic}(\widetilde{X}) \cong \operatorname{Pic}(\widetilde{V}) \cong \mathbb{Z} \oplus \mathbb{Z} \oplus \mathbb{Z}$ по теореме Лефшеца.

Лемма 4. Пусть $\Lambda$ и $\Xi-$ диизорь на $\mathbb{P}^{1} \times \mathbb{P}^{1}$, являющиеся дивизорами вырождения расслоений $\tau$ и $\eta$ соответственно. Тогда би-степени $\Lambda$ и $\Xi$ суть $(5,3 \mathrm{~m})$ u $(6,2 \mathrm{~m})$ соответственно.

ДОКАЗАТЕЛЬСТво. Несложные выгисления.

С другой стороны, в [10] доказан следующий результат.

Tеорема 5. Пусть $\omega: Y \rightarrow Z$ - расслоение на коники, $Y$ - неособое трехмерное многообразие, $\operatorname{Pic}(Y / Z)=\mathbb{Z}$, а поверхность $Z$ есть $\mathbb{F} r$ или $\mathbb{P}^{2}$. Тогда из рациональности трехмерного многообразия $Y$ следует $\left|2 K_{Z}+\Delta\right|=\varnothing$, где $\Delta-$ дивизор вырождения расслоения $\omega$.

Таким образом, из леммы 4 и теоремы 5 следует, что многообразия $\widetilde{X}$ и $\widetilde{V}$ нерациональны при $m \geqslant 2$. Значит, теорема 1 доказана (см. замечание 3 ). Следует отметить, что описанный метод несложно обобщается на многие неособые трехмерные многообразия, расслоенные на поверхности дель Пещо степени 1, 2 и 3. Для неособых трехмерных многообразий, расслоенных на поверхности дель Пецщо степени 4, вырождение не требуется, поскольку такие многообразия всегда представимы в виде расслоения на коники. В последнем случае вопрос рациональности исследован в [9]. Все трехмерные многообразия, расслоенные на поверхности дель Пещо степени не меньше 5 , рациональны (см. [10]).

\section{СПИСОК ЛИТЕРАТУРЫ}

[1] J. Kollár. Rational Curves on Algebraic Varieties. Berlin: Springer-Verlag, 1996. [2] В. А. Исковских // Труды МИАН. 1997. Т. 218. С. 190-232. [3] В. В. Шокуров // Изв. AH CCCP. Сер. матем. 1983. T. 23. № 4. C. 785-855. [4] F. Bardelli // Ann. Mat. Pura Appl. (4). 1984. V. 137. Р. 287-369. [5] А. В. Пухликов // Изв. РАН. Сер. матем. 1998. Т. 62. № 1. C. 123-164. [6] J. Kollár // London Math. Soc. Lecture Note Ser. 2000. V. 281. P. 51-71. [7] И. В. Соболев // Изв. РАН. Сер. матем. 2002. Т. 66. № 1. С. 203-224. [8] Т. Matsusaka // Nagoya Math. J. 1968. V. 31. P. 185-245; corrections, ibid. V. 33. P. 137; V. 36. P. 119. [9] В. А. Алексеев // Матем. заметки. 1987. Т. 41. № 5. С. 724-730. [10] В. А. Исковских // УMH. 1996. T. 51. № 4. C. 3-72. 